\title{
EFFET D'UN CHOC HYPOTONIQUE SUR LE VOLUME CELLULAIRE ET SUR LA CONCENTRATION INTRACELLULAIRE DE CALCIUM DES CELLULES BRANCHIALES DE TRUITE ARC-EN-CIEL.
}

\section{LEGUEN et P. PRUNET}

INRA, Laboratoire de Physiologie des Poissons, Campus de Beaulieu, 35042 Rennes Cedex, France.

\section{RÉSUMÉ}

La branchie étant un organe à structure complexe, il était impossible d'étudier l'effet d'un choc hypotonique sur les cellules branchiales in situ. Une culture primaire de branchie de truite a donc été mise au point et l'effet d'une dilution du milieu extracellulaire sur ces cellules en culture a été étudié.

Les cellules branchiales en culture primaire forment un épithélium à confluence après 5 à 7 jours de culture. Ces cellules présentent les caractéristiques morphologiques des cellules respiratoires de la branchie.

Les mesures du volume cellulaire, lors d'un choc hypotonique, sont effectuées sur des populations isolées de cellules respiratoires, à l'aide du système Coulter counter. Lors de la dilution aux 2/3 du milieu externe, les cellules gonflent pour atteindre un volume moyen de $130 \%$ en 5 minutes. Ensuite, les cellules régulent lentement leur volume. Après 30 minutes dans les conditions hypotoniques, le gonflement cellulaire ne représente plus que $110 \%$ du volume initial.

Lors d'un choc hypotonique, nous avons mesuré, à l'aide d'une sonde fluorescente sensible au calcium (le Fura-2), une augmentation de la concentration intracellulaire de calcium des cellules respiratoires en culture primaire.

Mots-clés : cellules branchiales, culture primaire, calcium intracellulaire, régulation du volume cellulaire.

\section{EFFECT OF HYPOTONIC SHOCK ON CELL VOLUME AND INTRACELLULAR CALCIUM OF TROUT GILL CELLS.}

\section{ABSTRACT}

Gill epithelium being a very complex structure, it was impossible to study the effect of an hypotonic shock on gill cells in situ. For this reason, a primary culture of trout gill cells was developed and the effect of the dilution of extracellular medium was measured on these gill cultured cells.

Gill cells in primary culture form a confluent monolayer of contiguous cells after 5 to 7 days of culture. These cultured cells display ultrastructural features resembling those of in situ respiratory cells. 
Measurements of cell volume, during a hypotonic shock, were made on isolated cultured respiratory cells with a Coulter counter technique. A hypotonic shock (2/3rd Ringer) produces a cell swelling of $130 \%$ in 5 minutes. Then, cells regulate their volume : after 30 minutes in hypotonic conditions, cell swelling represents $110 \%$ only of the initial value.

By measuring intracellular calcium with a fluorescent probe sensitive to calcium (Fura-2), we observed that a hypotonic shock produces an intracellular calcium increase in gill cultured cells.

Key-words : gill cells, primary culture, intracellular calcium, volume regulation.

\section{INTRODUCTION}

A l'interface entre milieu externe et milieu interne, la branchie joue un rôle majeur dans le maintien de la balance hydrominérale chez les poissons. En outre, cet organe joue aussi un rôle-clé dans la respiration ; la balance acide-base et l'excrétion de déchets azotés (McDONALD et al., 1989 ; GOSS et al., 1992 ; PIIPER et SCHEID, 1992). L'épithélium branchial est composé de deux types de cellules épithéliales : les cellules pavimenteuses ou respiratoires et les cellules riches en mitochondries ou cellules à chlorure. Les cellules respiratoires représentent 90 à $95 \%$ de l'épithélium et les cellules à chlorure représentent environ $5 \%$ des cellules branchiales.

De par leur fonction de transport (absorption de $\mathrm{NaCl}$ en eau douce et excrétion de sel en eau de mer) (EVANS, 1979), les cellules branchiales peuvent être sujettes à des variations de leur volume cellulaire, les transports de sels étant suivis d'un flux d'eau selon le gradient osmotique. De même, des modifications du volume peuvent apparaître lors du métabolisme organique des cellules. Contrairement aux mammifères où la pression osmotique plasmatique est bien régulée, il a été mesuré des variations significatives et durables de la pression osmotique du plasma de poissons. II en résulte des changements de la pression osmotique du milieu extracellulaire pouvant être aussi à l'origine des modifications du volume cellulaire. Ainsi, les cellules branchiales peuvent subir un rétrécissement cellulaire lors d'un transfert brutal de poisson d'eau douce en eau de mer où la pression osmotique plasmatique augmente d'environ 50\% (PRUNET et al., 1985). A l'inverse, les cellules peuvent gonfler lors d'un stress de confinement ou lors d'exercice, les niveaux de $\mathrm{Na}^{+}$et de $\mathrm{Cl}$ plasmatiques chutant de 10-20\% (McDONALD et ROBINSON, 1993 ; POSTLETHWAITE et MCDONALD, 1995). II est donc important pour les cellules branchiales de pouvoir réguler leur volume dans de telles situations, afin de survivre et de maintenir leurs fonctions. Ainsi, lors d'un gonflement cellulaire, les cellules doivent mettre en place des mécanismes de régulation du volume.

Dans la plupart des types cellulaires, les cellules gonflées régulent leur volume en perdant des ions potassium, des ions chlore et parfois des composés organiques. Cette perte de sels s'accompagne d'une perte d'eau, c'est le phénomène de régulation du volume après un gonflement cellulaire (RVD : Regulatory Volume Decrease) (HOFFMANN et SIMONSEN, 1989 ; SARKADI et PARKER, 1991 ; SPRING et HOFFMANN, 1992 ; HOFFMANN et DUNHAM, 1995).

De nombreuses études montrent un rôle majeur du calcium comme signal de transduction de la régulation du volume après un gonflement cellulaire. Le calcium intracellulaire active, dans plusieurs types cellulaires, la sortie de sels et de composés organiques lors d'un choc hypotonique (PIERCE et POLITIS, 1990 ; McCARTY et O'NEIL, 1992 ; FOSKETT, 1994). 
Dans notre étude, nous avons mesuré la capacité des cellules branchiales à réguler leur volume et la réponse calcique suite à un choc hypotonique. En raison de la complexité structurale de la branchie, il n'était pas possible d'étudier sur le tissu en place la régulation du volume cellulaire ni l'évolution du calcium intracellulaire lors d'un choc hypotonique. Par conséquent, nous avons développé une technique de culture primaire de cellules branchiales de truite arc-en-ciel (Oncorhynchus mykiss).

\section{MATÉRIEL ET MÉTHODES}

\section{La culture primaire}

La technique de culture primaire développée dans notre étude est dérivée de celle décrite par PÄRT et al., 1993.

Des truites arc-en-ciel (Oncorhynchus mykiss) immatures sont anesthésiées, puis une perfusion cardiaque est effectuée afin d'éliminer les hématies présentes dans les branchies. Les arcs branchiaux sont prélevés puis rincés. Ensuite, les cellules branchiales sont isolées par traitement à la trypsine. Après plusieurs lavages, les cellules sont ensemencées dans des boîtes de culture à une densité de 4 à $5^{\star} 10^{6}$ cellules par $\mathrm{cm}^{2}$. Le milieu de culture se compose de L15 supplémenté avec $5 \%$ de sérum de veau foetal, $2 \mathrm{mM}$ de glutamine, $0.5 \mu \mathrm{g} / \mathrm{ml}$ d'amphotéricine $\mathrm{B}, 100 \mathrm{Ul} / \mathrm{ml}$ de pénicilline, $100 \mu \mathrm{g} / \mathrm{ml}$ de streptomycine avec un $\mathrm{pH}$ final de 7.6 et une pression osmotique entre 300 et $310 \mathrm{mOsm} / \mathrm{l}$. Les cultures sont maintenues dans un incubateur à $18^{\circ} \mathrm{C}$. Le milieu est changé 24 heures après ensemencement, puis tous les deux jours. Les mesures de volume cellulaire et de la concentration intracellulaire de calcium sont effectuées sur des cellules ayant entre 5 à 12 jours de culture.

\section{Mesure du volume cellulaire}

Les mesures du volume cellulaire ont été effectuées sur des populations de cellules branchiales cultivées puis isolées par traitement à la trypsine. L'évolution du volume moyen des cellules au cours du temps est mesurée électriquement à l'aide de l'appareil Coulter counter connecté à un ordinateur. Un programme développé par Coulter est utilisé pour évaluer le volume cellulaire moyen d'une population de cellules comptées. Ce volume moyen en unité arbitraire est calculé comme la médiane des courbes de distributions du volume cellulaire. Les volumes cellulaires absolus sont obtenus en utilisant des billes de latex comme standard. Les changements de volume sont présentés en volume relatif $\left(\mathrm{V} / \mathrm{V}_{0}\right)$ par rapport à un volume initial mesuré dans un milieu isosmotique.

\section{Mesure de la concentration intracellulaire de calcium}

Les mesures de la concentration intracellulaire de calcium dans les cellules branchiales en culture primaire sont effectuées à l'aide d'une méthode fluorimétrique utilisant les variations de fluorescence d'une sonde sensible au calcium : le Fura-2. Le Fura-2 n'est pas liposoluble, donc ne traverse pas spontanément la bicouche lipidique membranaire des cellules. L'incorporation de la sonde fluorescente dans les cellules nécessite l'emploi d'une forme estérifiée, le Fura-2-pentaacétoxyméthyl ester (Fura-AM) qui est lipophile. Une fois incorporé dans les cellules, le Fura-AM est hydrolysé sous l'action des estérases cytoplasmiques et se retrouve sous sa forme désestérifiée, piégé dans le cytoplasme. Les cellules cultivées sont chargées avec la sonde fluorescente en les incubant en présence de $4 \mu \mathrm{mol} / /$ de Fura-2AM pendant 30 minutes. 
Les cellules chargées sont placées sur la platine d'un microscope. Un monochromateur d'excitation disposé entre la lampe à xénon et le microscope permet de sélectionner successivement les deux longueurs d'excitation pour le Fura (352 et $380 \mathrm{~nm}$ ). La lumière d'excitation est réfléchie vers les cellules grâce à un miroir dichroique. La lumière émise, suite à l'excitation, traverse un filtre d'arrêt ne laissant passer que les longueurs d'onde supérieures à $510 \mathrm{~nm}$. La lumière passe ensuite par un photomultiplicateur. Le signal émis par ce dernier est digitalisé et stocké sur le disque dur de l'ordinateur. Pour nos mesures, nous sélectionnons un groupe d'une dizaine de cellules branchiales.

Le logiciel "Felix " développé par PTI transforme les valeurs de fluorescence émises en concentration de calcium cytoplasmique à partir de l'équation suivante : $\left[\mathrm{Ca}^{+}\right]_{1}=$ $\mathrm{K}^{\prime} \times(\mathrm{R}-\mathrm{Rmin}) /(\mathrm{Rmax}-\mathrm{R})$ où $\mathrm{K}^{\prime}$ correspond au produit de la constante de dissociation du complexe $\mathrm{Ca}^{2+} /$ Fura-2 et de la constante liée aux caractéristiques optiques du système de fluorescence utilisé. R est le rapport des signaux de fluorescence mesuré à $352 \mathrm{~nm}$ et $380 \mathrm{~nm}$. Ce rapport est calculé après qu'une valeur de bruit de fond ait été soustraite à chaque longueur d'onde. Rmin et Rmax sont les valeurs de $R$ en absence de calcium et en présence de quantité saturante de calcium, respectivement. $K^{\prime}$, Rmin et Rmax sont calculés en utilisant une sonde Fura-2 désestérifié, encore appelé Fura acide, dans une gamme de tampon contenant des concentrations croissantes de calcium.

\section{RÉSULTATS ET DISCUSSION}

\section{La culture primaire}

Après 5 à 7 jours de culture, nous obtenons une monocouche de cellules branchiales à confluence. En microscopie optique, ces cellules apparaissent plates, de forme épithéliale et mesurant entre 10 et $50 \mu \mathrm{m}$. L'analyse ultrastructurale des cellules branchiales montre que la très grande majorité des cellules en culture présentent les caractéristiques des cellules pavimenteuses in situ encore appelées cellules respiratoires (LEGUEN et al., 1997). La présence dans les cellules en culture d'un revêtement (“ coat ») apical, de jonctions cellulaires apicales de type serré et de microcrêtes sur leur membrane apicale témoignent d'un maintien de polarité indispensable au fonctionnement des cellules. La présence d'un abondant réticulum endoplasmique et d'un appareil de Golgi étendu indique que ces cellules en culture présentent une grande activité métabolique.

En accord avec les résultats de cultures primaires développées à partir de branchie de truite (PÄRT et al., 1993) et de bar (AVELLA et al., 1994), nous n'avons pu maintenir les cellules à chlorure en culture. Néanmoins, la culture représente un bon modèle pour étudier la physiologie des cellules respiratoires in vitro. Des travaux récents montrent que ces cellules en culture régulent leur $\mathrm{pH}$ intracellulaire à l'aide d'un échangeur $\mathrm{Na} / \mathrm{H}^{+}$ (PÄRT et WOOD, 1996) et que cette régulation diffère selon que les cellules cultivées sont issues de truites élevées en eau douce ou adaptées à l'eau de mer (LEGUEN et al., 1997). Nous avons donc utilisé ce système in vitro pour mesurer l'effet d'un choc hypotonique sur le volume cellulaire et sur la concentration intracellulaire en calcium de ces cellules respiratoires.

\section{Effet d'un choc hypotonique sur le volume cellulaire}

Lors de la dilution du milieu externe aux $2 / 3$, les cellules branchiales en culture primaire gonflent pour atteindre un volume moyen de $130 \%$ en 5 minutes. Ensuite, les cellules régulent lentement leur volume. Après 30 minutes dans les conditions 
hypotoniques, le gonflement cellulaire ne représente plus que $110 \%$ du volume initial (figure 1). Cette cinétique de régulation du volume est identique à ce qui a été observé dans plusieurs types cellulaires dont la lignée $A 6$ issue de rein d'amphibien (EHRENFELD et al., 1994 ; DE SMET et al., 1995), les cellules du tubule proximal de Malpighi (ARENSTEIN et al., 1995), les cellules de la glande lacrymale (PARK et al., 1994). La régulation du volume des cellules branchiales lors d'un choc hypotonique doit se faire probablement par la perte de $\mathrm{K}^{+}$et de $\mathrm{Cl}$, accompagnée d'une sortie d'eau selon le gradient osmotique, comme il a été montré dans de nombreux types cellulaires dont les hépatocytes de truites (BIANCHINI et al., 1988). Cette régulation du volume peut être aussi associée à la perte de composé organique, mise en évidence dans les cellules d'organismes exposés fréquemment à des changements de leur osmolarité externe comme les animaux marins ou vivant dans les estuaires (PIERCE et POLITIS, 1990). II serait intéressant de confirmer ces mécanismes de régulation du volume dans les cellules branchiales.

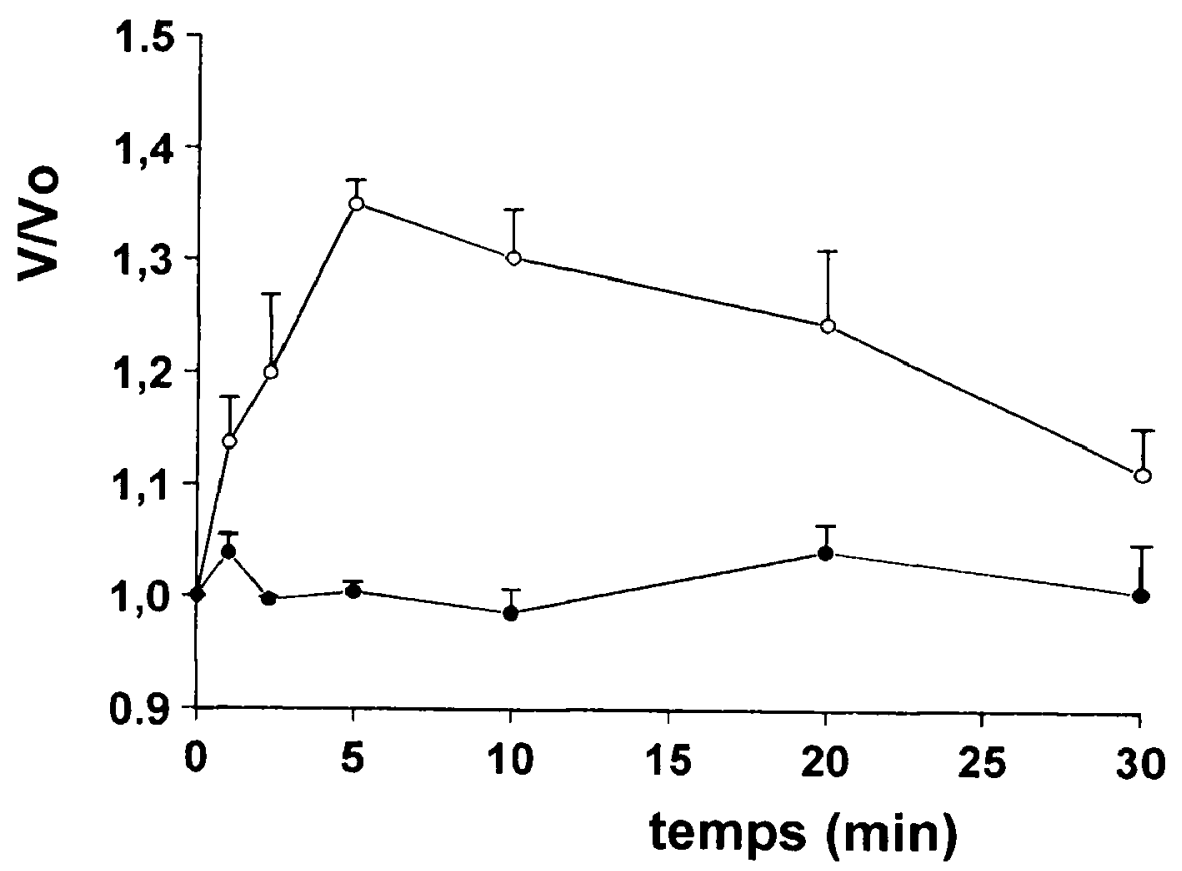

Figure 1

Effet d'un choc hypotonique sur le volume cellulaire de cellules branchiales en culture.

Évolution du volume cellulaire mesuré par Coulter counter, au cours du temps, dans les conditions contrôles (Ringer) $(\cdot)$ et après un choc hypotonique (dilution du Ringer aux 2/3) provoqué au temps zéro ( $O$ ). Les cellules utilisées sont des cellules branchiales cultivées issues de truites élevées en eau douce et elles sont isolées par traitement à la trypsine. Les valeurs représentent les moyennes de 4 expériences. Les barres verticales représentent les SEM (erreur standard à la moyenne).

Figure 1

Effect of hypotonic shock on cell volume of trout cultured gill cells.

Measure of the evolution of cell volume, by Coulter counter technique, in control conditions (Ringer) $(\cdot)$ and after a hypotonic shock (2/3rd dilution) produced at time zero $(O)$. Gill cultured cells from freshwater trout were isolated by trypsinization. Values are means $\pm \operatorname{SEM}(n=4)$. 


\section{Effet d'un choc hypotonique sur la concentration intracellulaire de calcium}

Lors d'un choc hypotonique, une augmentation rapide de la concentration intracellulaire de calcium est enregistrée dans les cellules branchiales en culture primaire. Comme nous l'observons sur la figure 2 représentant l'effet d'un choc hypotonique sur un groupe de cellules, la dilution du milieu externe induit un pic transitoire de la concentration de calcium intracellulaire (pic de $106 \pm 10 \mathrm{nM}$ à $269 \pm 23 \mathrm{nM}, \mathrm{n}=16$ ) qui a lieu 20 à $30 \mathrm{~s}$ après le début de la perfusion du Ringer dilué. Le pic de $\mathrm{Ca}^{2}$ est suivi d'un plateau correspondant à un niveau de calcium intracellulaire significativement supérieur, de 152.5 $\pm 13 n M(n=16)$, au niveau de base. Cette deuxième phase en plateau se maintient durant toute la période d'incubation des cellules en condition hypotonique. Le retour à la concentration intracellulaire initiale se fait en quelques secondes après la perfusion du Ringer normal $(114 \pm 11 \mathrm{nM}, \mathrm{n}=16)$. Les variations de la concentration intracellulaire de calcium lors d'un choc hypotonique suggèrent que cet ion joue un rôle important dans la régulation du volume des cellules branchiales comme pour de nombreux types cellulaires (PIERCE et POLITIS, 1990 ; McCARTY et O'NEIL, 1992). Dans plusieurs études, il a été montré que le calcium intracellulaire agissait comme signal de transduction de la régulation du volume après un gonflement cellulaire, il active la sortie de $\mathrm{K}^{\prime}, \mathrm{Cl}$ (CALA, 1983 ; PARK et al., 1994) et de composés organiques (AMENDE et PIERCE, 1980 ;

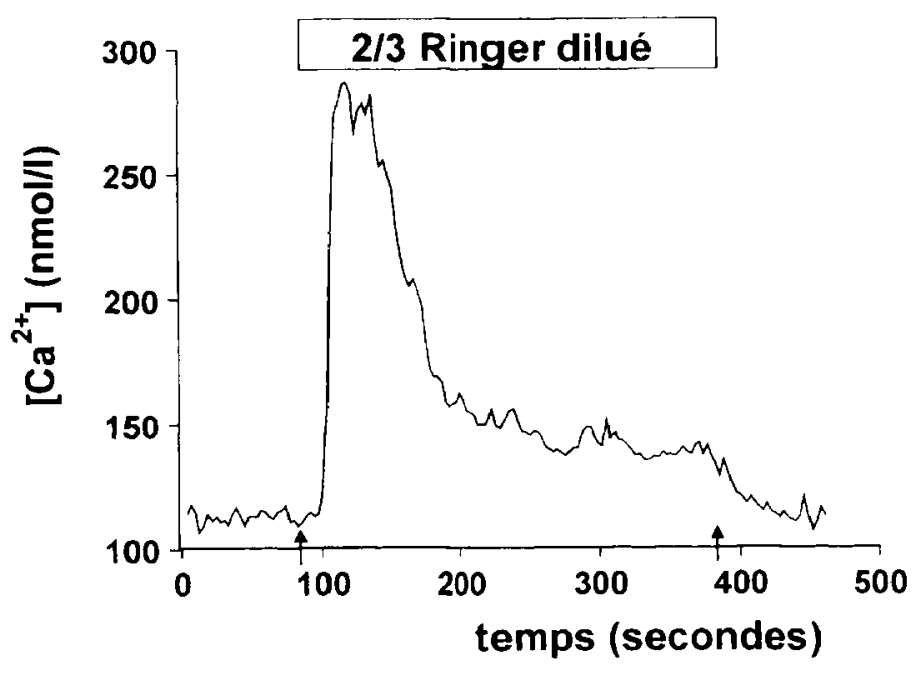

Figure 2

Effet d'un choc hypotonique sur la concentration intracellulaire de calcium dans les cellules branchiales en culture.

Figure représentative de l'évolution de la concentration intracellulaire de calcium au cours du temps lors d'un choc hypotonique (dilution du Ringer aux 2/3). La mesure de la concentration intracellulaire est effectuée à l'aide d'une sonde fluorescente sensible au calcium, le Fura-2. Les cellules utilisées sont des cellules branchiales cultivées issues de truites élevées en eau douce. Cette figure a été effectuée sur un groupe de 10 cellules branchiales.

Figure 2

Effect of hypotonic shock on intracellular calcium concentration of trout cultured gill cells.

Representative figure of the evolution of intracellular calcium concentration during a hypotonic shock (2/3rd dilution). Intracellular calcium measurements were obtained with a Ca-sensitive fluorescent probe : Fura-2. Cultured cells used are gill cells from freshwater trout. This figure is the response of 10 gill cells. 
BEVAN et al., 1990). Des expériences préliminaires effectuées en présence ou en absence de calcium dans le milieu extracellulaire suggèrent que cette élévation de calcium cytoplasmique serait due, d'une part, à une entrée de calcium et, d'autre part, à la mobilisation de réserves calciques c'est-à-dire à la sortie du calcium du réticulum endoplasmique.

Nos résultats confirment la présence d'un mécanisme de régulation du volume dans les cellules branchiales de truite comme dans les autres types cellulaires. Ces cellules doivent éviter des variations de leur volume afin de maintenir leurs fonctions qui sont capitales pour la survie du poisson. Nous avons montré dans notre étude qu'une augmentation de la concentration intracellulaire de calcium avait lieu lors du gonflement cellulaire. Cette augmentation précédant la régulation du volume, ceci suggère l'implication du calcium intracellulaire dans le déclenchement de cette régulation. II sera important maintenant de chercher à caractériser les mécanismes impliqués dans les modifications du calcium intracellulaire. L'analyse et la compréhension de ces mécanismes sont d'autant plus importantes que toute perturbation de l'homéostasie calcique intracellulaire, que ce soit en relation avec la présence de polluant ou avec des situations physiologiques particulières, est susceptible de conduire à des problèmes de régulation du volume de ces cellules et donc de perturber les fonctions physiologiques associées aux cellules respiratoires (échanges de gaz, d'ions).

\section{BIBLIOGRAPHIE}

AMENDE L.M. and PIERCE S.K., 1980. Free amino acid mediated volume regulation of isolated Noetia ponderosa red blood cells : control by $\mathrm{Ca}^{2+}$ and ATP. J. Comp. Physiol., 138, 291-298.

ARENSTEIN I.R., CARUSO-NEVES C., ONUCHIC L.F. and LOPES A.G., 1995. Mechanisms of cell volume regulation in the proximal segment of the malpighian tubule of Rhodnius neglectus. J. membrane Biol., 146, 47-57.

AVELLA M., BERHAUT J. and PAYAN P., 1994. Primary culture of gill epithelial cells from the sea bass Dicentrarchus labrax. In Vitro Cell Dev. Biol., 30A, 41-49.

BEVAN C., THEISS C. and KINNE R.K.H., 1990. Role of Ca+ in sorbitol release from rat inner medullary collecting duct (IMCD) cells under hypoosmotic stress. Biochem. Biophys. Res. Commun., 170, 563-568.

BIANCHINI L., FOSSAT B., PORTHÉ-NIBELLE J., ELLORY J.C. and LAHLOU B., 1988. Effects of hypoosmotic shock on ion fluxes in isolated trout hepatocytes. J. Exp. Biol., 137, 303-318.

CALA P.M., 1983. Cell volume regulation by Amphiuma red blood cells. The role of $\mathrm{Ca}^{2+}$ as a modulator of alkali metal/ $\mathrm{H}^{+}$exchange. J. Gen. Physiol., 82, 761-784.

DE SMET P., SIMAELS J. and VAN DRIESSCHE W., 1995. Regulatory volume decrease in a renal distal tubular cell line (A6). I.-Role of $\mathrm{K}^{+}$and $\mathrm{Cl}^{-}$. Pflügers Arch., 430, 936944.

EHRENFELD J., RASCHI C. and BROCHIERO E., 1994. Basolateral potassium membrane permeability of $A 6$ cells and cell volume regulation. J. Membr. Biol., 138, 181-195.

EVANS D.H., 1979. Fish. In : Comparative physiology of osmoregulation in animals, edited by G.M.O. Maloiy, vol. 1, 305-390.

FOSKETT J.K., 1994. The role of calcium in the control of volume regulatory transport pathways. In : Cellular and Molecular Physiology of cell volume regulation, edited by Strange K., CRC Press, Boca Raton (FL.), 259-277. 
GOSS G.G., PERRY S.F., WOOD C.M. and LAURENT P., 1992. Mechanisms of ion and acid-base regulation at the gills of freshwater fish. J. Exp. Zool., 263, 143-149.

HOFFMANN E.K. and SIMONSEN L.O., 1989. Membrane mechanisms in volume and $\mathrm{pH}$ regulation in vertebrate cells. Physiol. Rev., 69, 315-382.

HOFFMANN E.K. and DUNHAM P.B., 1995. Membrane mechanisms and intracellular signalling in cell volume regulation. Int. Rev. Cyt., 161, 173-262.

LEGUEN I., PISAM M., BIDET M., TAUC M. and POUJEOL P., 1998. pHi regulation and ultrastructural analysis in cultured gill cells from freshwater or seawater-adapted trout. Fish Physiol. Biochem., 18, 297-309.

McCARTY N.A. and O'NEIL R.G., 1992. Calcium signalling in cell volume regulation. Physiol. Rev., 72, 1037-1061.

MCDONALD D.G., TANG Y. and BOUTILIER R.G., 1989. Acid and ion transfer across the gills of fish : mechanisms and regulation. Can. J. Zool., 67, 3046-3054.

MCDONALD D.G. and ROBINSON J.G., 1993. Physiological responses of lake trout to stress : effects of water hardness and genotype. Transaction of the American Fisheries Society, 122, 1146-1155.

PARK K.P., BECK J.S., DOUGLAS I.J. and BROWN P.D., 1994. Ca(2+)-activated K+ channels are involved in regulatory volume decrease in acinar cells isolated from the rat lachrymal gland. J. Membr. Biol., 141, 193-201.

PÄRT P., NORRGREN L., BERGSTRÖM E. and SJÖBERG P., 1993. Primary cultures of epithelial cells from rainbow trout gills. J. Exp. Biol., 175, 219-232.

PÄRT P. and WOOD C.M., 1996. Na/H exchange in cultured epithelial cells from fish gills. J. Comp. Physiol. B, 166 (1), 37-45.

PIERCE S.K. and POLITIS A.D., 1990. $\mathrm{Ca}^{2 *}$-activated cell volume recovery mechanisms. Annu. Rev. Physiol., 52, 27-42.

PIIPER J. and SCHEID P., 1992. Gas exchange in vertebrates through lungs, gills, and skin. N.I.P.S., 7, 199-203.

POSTLETHWAITE E.K. and MCDONALD D.G., 1995. Mechanisms of $\mathrm{Na}^{\circ}$ and $\mathrm{Cl}$ regulation in freshwater-adapted rainbow trout (Oncorhynchus mykiss) during exercise and stress. J. Exp. Biol., 198, 295-304.

PRUNET P., BOEUF G. and HOUDEBINE L.M., 1985. Plasma and pituitary prolactin levels in rainbow trout during adaptation to different salinities. J. Exp. Zool., 235, 187-196.

SARKADI B. and PARKER J.C., 1991. Activation of ion transport pathways by changes in cell volume. Biochim. Biophy. Acta, 1071, 407-427.

SPRING K.R. and HOFFMANN E.K., 1992. Cell volume control. In : Seldin D.W. and Giebish G. (eds.), The kidney : Physiology and Pathophysiology, 2nd edition, chapter 6 , 147-169, New York, Raven Press. 